\title{
Acute effects of tobacco smoke on human airway dendritic cells in vivo
}

\author{
M. Lommatzsch, K. Bratke, T. Knappe, A. Bier, K. Dreschler, M. Kuepper, P. Stoll, \\ P. Julius and J.C. Virchow
}

ABSTRACT: Airway dendritic cells (DCs) play a key role in smoke-related lung diseases; however, the acute effects of tobacco smoke on human airway DCs in vivo are unknown.

A total of 16 smokers underwent bronchoalveolar lavage at two time-points: directly after a 4-h period of nonsmoking (no smoke exposure); and directly after a 4-h period during which eight cigarettes were smoked (acute smoke exposure). Using flow cytometry, myeloid DCs (mDCs) and plasmacytoid DCs (pDCs), as well as function-associated surface molecules on mDCs, were analysed in bronchoalveolar lavage fluid (BALF) and in blood.

The numbers of macrophages, lymphocytes, neutrophils, eosinophils and pDCs were unchanged in BALF following acute smoke exposure, as compared to no smoke exposure. In contrast, there was a strong increase in $\mathrm{mDC}$ number in BALF and a concomitant decrease in mDC number in blood following acute smoke exposure. In addition, acute smoke exposure led to an increase in the expression of the surface molecules blood dendritic cell antigen 1 and 4 and a decrease in the expression of the lung homing receptor, $\mathrm{CC}$ chemokine receptor 5 , on $\mathrm{mDCs}$ in BALF.

Acute tobacco smoke inhalation results in an immediate and selective recruitment of mDCs into human airways, which might reflect the very early reaction of the adaptive immune system to smoke exposure.

KEYWORDS: Bronchoalveolar lavage fluid, dendritic cells, flow cytometry, tobacco smoke

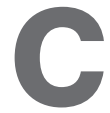

igarette smoking is the main risk factor for the development of inflammatory lung diseases such as chronic obstructive pulmonary disease (COPD) [1, 2] or pulmonary Langerhans' cell histiocytosis (PLCH) [3, 4]. The mechanisms leading to these diseases remain incompletely understood [5]. However, the accumulation of dendritic cells (DCs) and lymphocytes within the lung, and their association with disease severity, suggest that adaptive immune responses play a role in these diseases [4-9]. Airway DCs initiate and control adaptive immune responses in the lung [10]. Following antigen uptake in the airways [11], DCs migrate to the draining lymph nodes in order to present antigenic information to specialised lymphocytes, which organise an inflammatory response against the encountered antigen [10]. In addition, DCs can also present antigens locally to lymphocytes within the lung parenchyma $[9,12]$.

Chronic cigarette smokers display a characteristic increase in the number of Langerhans' cells, a subtype of myeloid DCs (mDCs), in the airways [13-15]. Even more Langerhans' cells are found in the airways of smokers with COPD [6] or
PLCH [3, 16]. However, the significance of the Langerhans' cell phenotype in the pathogenesis of these diseases remains unknown. Using a recently described flow cytometric method for analysing DCs in human bronchoalveolar lavage fluid (BALF) [17, 18], the expression of functionassociated surface molecules on airway mDCs were characterised in chronic cigarette smokers [15]. The airway mDCs of smokers display increased expression of the co-stimulatory molecules CD80 and CD86, but reduced expression of the lymph node homing receptor, CC chemokine receptor (CCR) 7 [15]. These findings led to the hypothesis that mDCs of smokers might show increased ability to induce T-cell responses, but reduced ability to migrate to draining lymph nodes [15].

In contrast to the effects of chronic tobacco smoke exposure, there is currently no information regarding the acute effects of tobacco smoke on airway DCs in vivo. Animal models exploring airway DCs have used protocols with tobacco smoke exposures of $\geqslant 1$ day [19]. Human studies exploring the acute effects of smoking on pulmonary immune cells did not investigate
AFFILIATIONS

Dept of Pneumology, University of

Rostock, Rostock, Germany.

CORRESPONDENCE

M. Lommatzsch

Abteilung für Pneumologie

Klinikund Poliklinik für Innere

Medizin I

Universität Rostock

Ernst-Heydemann-Str. 6

18057 Rostock

Germany

E-mail: marek.lommatzsch@

med.uni-rostock.de

Received:

June 072009

Accepted after revision:

Sept 012009

First published online:

Sept 092009 
airway DCs [20, 21]. It was the aim of the present study, therefore, to investigate the effects of acute tobacco smoke exposure on the number and surface molecule expression of human airway DCs in vivo. Using the above-mentioned flow cytometric method [17, 18], mDCs and plasmacytoid DCs (pDCs) in BALF directly after a 4-h period of acute smoke exposure were analysed and the results compared intraindividually with parameters obtained directly following a 4-h period of smoking abstinence. This early time-point of analysis was chosen in order to test for a possible rapid reaction of the adaptive immune system in the airways. This decision was based on previous data from McWilliam et al. [22], who described a rapid influx of DCs into the airways of rats, peaking as soon as $2 \mathrm{~h}$ after antigen exposure.

\section{METHODS}

\section{Participants}

The participants were recruited in Rostock (Germany), using the following inclusion criteria: 1) age of 20-50 yrs, and 2) current smoker of $\geqslant 10$ cigarettes $\cdot$ day $^{-1}$. Exclusion criteria were as follows: 1) any history of chronic diseases, 2) any regular medication, and 3) any signs of a respiratory tract infection within the 2 weeks prior to bronchoscopy. The study was approved by the Ethics Committee of the Mecklenburg-Vorpommern General Medical Council (Rostock, Germany). The study was registered at ClinicalTrials.gov (trial number NCT00740896). All participants provided written informed consent.

\section{Study design}

All participants were examined in the Dept of Pneumology (University of Rostock, Rostock, Germany) at two time-points (A and B), with a 3-week interval in between. At both timepoints, participants were permitted to smoke ad libitum until 07:00 h (fig. 1). At time-point A (no smoke exposure), the participants refrained from smoking between 07:00 and 11:00 h. At time-point B (acute smoke exposure), participants smoked eight cigarettes between 07:00 and 11:00 h. At both time-points, the participants underwent bronchoscopy (with bronchoalveolar lavage) between 11:00 and 12:00 h. In order to exclude the possibility that the bronchoscopy sequence would influence any of the parameters measured in the BALF, a crossover design was chosen. Time-point A was followed by time-point B in the first group (eight participants), whereas this sequence was reversed in the second group (eight participants) (fig. 1). The participants were randomised into the two groups of the study. Directly before each bronchoscopic procedure, lung function was assessed using body plethysmography (MasterScreen; Jaeger, Viasys, Hoechberg, Germany). Then, a venous cannula was placed and $10 \mathrm{~mL}$ blood were taken for laboratory analyses and for the quantification of DCs in blood. Afterwards, bronchoscopy was performed, with inhalation (PARI BOY; PARI, Starnberg, Germany) of $4 \%$ lidocaine for $15 \mathrm{~min}$ prior to the procedure. The analyst of the flow cytometric data (K. Bratke) was blinded to the exposure assignment of the participants. The clinical investigators who supervised the participants and performed the blood collection and bronchoscopy procedures were informed about the intervention.

\section{Bronchoalveolar lavage, flow cytometry and ELISA}

Bronchoalveolar lavage was performed using flexible bronchoscopes (Olympus, Hamburg, Germany). The bronchoscope was wedged into a subsegment of the right middle lobe and a total of $100 \mathrm{~mL}$ pre-warmed sterile saline was instilled. The fluid was recovered by gentle aspiration. BALF cells were isolated and counted as described previously $[17,18]$. DCs in BALF were analysed using four-colour flow cytometry, as previously described [17, 18], and the antibody panel detailed in table 1. Blood DCs were analysed in freshly collected EDTA-blood using a similar approach $[17,18]$. Dead cells were excluded from the DC analyses because the initial gating step (which excludes highly fluorescent lineage-positive cells) excludes highly autofluorescent cells, such as dead cells [17, 18]. The surface molecule expression on mDCs was quantified using histograms as described previously $[17,18]$. The chemokines CC chemokine ligand (CCL) 2, 3, 5 and 20 and CXC chemokine ligand (CXCL) 10 and 12 were analysed in cell-free BALF supernatants using commercially available ELISAs according to the manufacturer's instructions (DuoSet; R\&D Systems, Minneapolis, MN, USA).

\section{Statistical analysis}

Data were analysed using SPSS (SPSS, Inc., Chicago, IL, USA). Most parameters were not normally distributed. Therefore, they were calculated as median (range). The comparison of parameters between time-points A (smoking cessation) and $\mathrm{B}$ (acute smoke exposure) was performed using the Wilcoxon test for related samples. Probabilities of $\mathrm{p}<0.05$ were regarded as significant.

\section{RESULTS}

\section{Characteristics of the participants}

A total of 16 current smokers (11 males and five females) were included in the present study based on the inclusion and exclusion criteria. The median (range) age was 27 (23-49) yrs, and the body mass index $23.5(18.6-29.8) \mathrm{kg} \cdot \mathrm{m}^{-2}$. The participants smoked 13 (10-30) cigarettes $\cdot$ day $^{-1}$, and the duration of smoking was 12 (4-33) yrs. This resulted in a smoking history of 7 (2-35) pack-yrs. The lung function parameters of the participants (pre-bronchodilator values) are given in table 2 . There was no significant difference in any of the lung function parameters between the two time-points (table 2). Three (19\%) out of 16 participants had a pre-bronchodilator forced expiratory volume in $1 \mathrm{~s} /$ forced vital capacity ratio of $<70 \%$ (at both time-points), but did not report signs or symptoms of COPD.

\section{Differential cell counts in blood and BALF}

The total and differential leukocyte counts in blood and BALF are detailed in table 2. There was no significant difference in total leukocyte counts or the percentages of macrophages, lymphocytes, neutrophils and eosinophils in BALF between the two time-points (table 2). In blood, total leukocyte counts and the percentages of lymphocytes and neutrophils did not differ between the time-points. In contrast, there was a significant decrease in the percentages of monocytes and eosinophils in peripheral blood following acute smoke exposure (table 2).

\section{DC counts in blood and BALF}

The percentage and total number of mDCs and pDCs in BALF and blood are detailed in table 3 . The number of $\mathrm{mDCs}$, but not pDCs, were increased in BALF and decreased in blood following acute smoke exposure, as compared to no smoke 


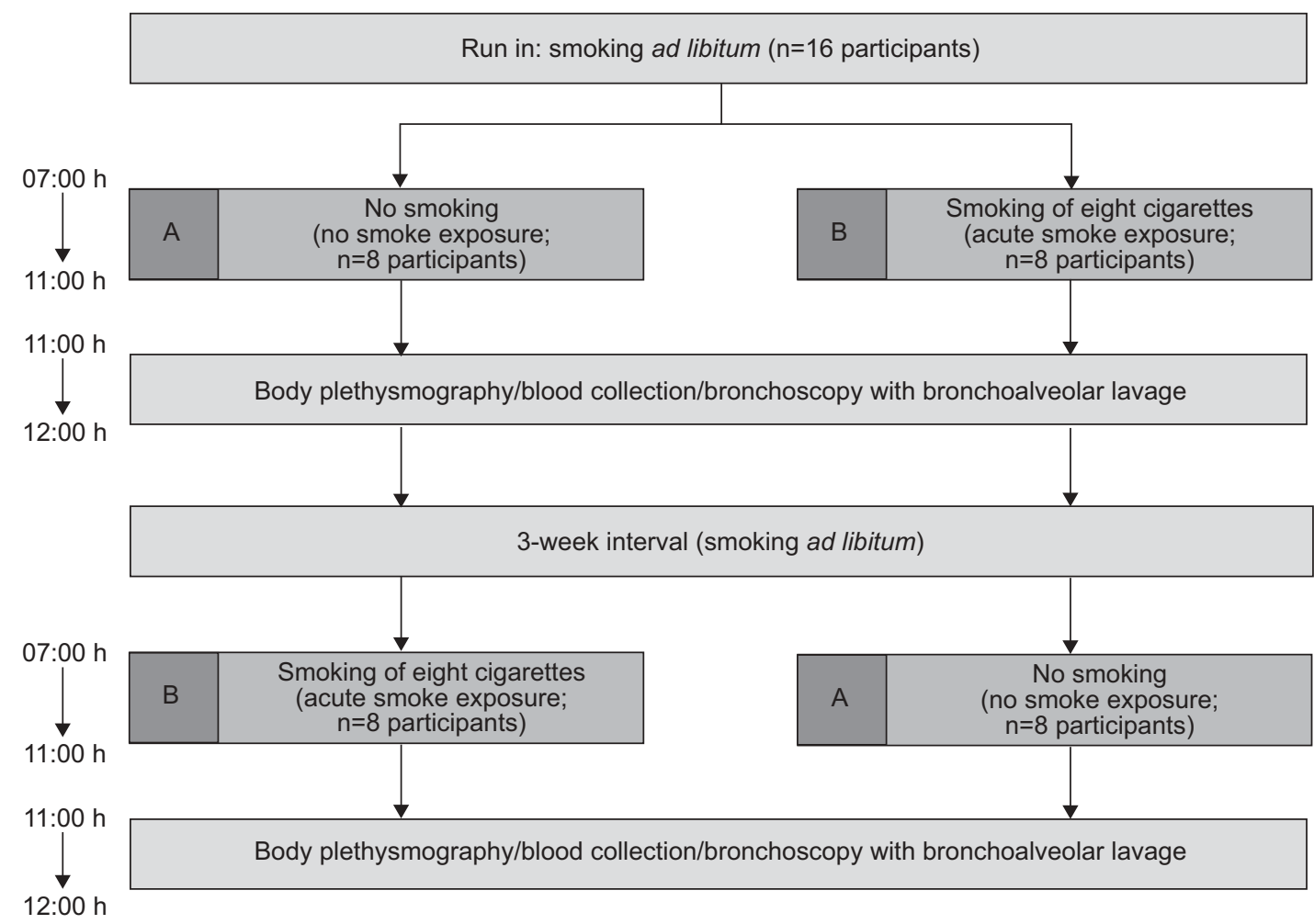

FIGURE 1. Study design. Participants were permitted to smoke ad libitum until $07: 00 \mathrm{~h}$ at the first time-point, and during the 3-week interval between the time-points. Time-point A (no smoke exposure for $4 \mathrm{~h}$ ) was followed by time-point B (acute smoke exposure for $4 \mathrm{~h}$ ) in the first group ( $\mathrm{n}=8$ participants), whereas this sequence was reversed in the second group ( $\mathrm{n}=8$ participants). At both time-points, participants underwent body plethysmography, blood collection and bronchoscopy (with bronchoalveolar lavage) between 11:00 and 12:00 $\mathrm{h}$.

exposure (table 3; fig. 2). It should be noted that the two participants with the highest numbers of mDCs in BALF $\left(>4,000 \mathrm{mDCs} \cdot \mathrm{mL} \mathrm{BALF}^{-1}\right.$ after $4 \mathrm{~h}$ of smoking cessation) did not show a further increase in BALF mDC numbers following acute smoke exposure (fig. 2).

\section{Chemokines in BALF}

In order to identify potential mechanisms leading to DC recruitment into the airways following smoke exposure, chemokines known to be involved in DC attraction were analysed in BALF. There was no significant difference in the BALF concentrations of CCL20 (macrophage inflammatory protein (MIP)-3 $\alpha$ ) and CXCL10 (interferon- $\gamma$-inducible protein 10) between time-points A and B (table 3). Concentrations of the chemokines CCL2 (monocyte chemotactic protein-1), CCL3 $(\mathrm{MIP}-1 \alpha)$, CCL5 (regulated on activation, normal T-cell expressed and secreted (RANTES)) and CXCL12 in BALF were below the respective detection limit in the majority of the samples, and could, therefore, not be evaluated (data not shown).

\section{Function-associated surface molecules on BALF mDCs}

The low median number of pDCs $\left(42-100\right.$ cells $\left.\cdot \mathrm{mL} \mathrm{BALF}^{-1}\right)$ (table 2) precluded a statistically reliable flow cytometric quantification of pDC surface molecules in BALF, and was, therefore, not performed. In contrast, a panel of functionassociated surface molecules could be analysed on mDCs in all participants at both time-points (table 4). There was a significant increase in the expression of blood dendritic cell antigen (BDCA) 1 (CD1c) and BDCA-4 (neuropilin-1), but no significant change in the expression of CD1a and langerin on BALF mDCs following acute smoke exposure, as compared to no smoke exposure (table 4). Expression of the lung homing receptor CCR5 on BALF mDCs was significantly decreased following acute smoke exposure. There was a nonsignificant trend towards increased expression of the macrophage mannose receptor (MMR; CD206) on BALF mDCs after acute smoke exposure. Expression of the co-stimulatory molecules CD80 and CD86, the lymph node homing receptor CCR7 and the maturity marker CD83 did not differ significantly between the time-points (table 4).

\section{DISCUSSION}

The present study is the first to identify the acute effects of tobbaco smoke exposure on the adaptive immune system in human airways in vivo. It was shown that there is immediate and selective recruitment of $\mathrm{mDCs}$, but not $\mathrm{pDCs}$, into the airways within hours following acute tobacco smoke exposure. In addition, specific changes in the expression of functionassociated surface molecules on airway mDCs were demonstrated following acute tobacco smoke exposure. Thus the present in vivo study provides important new information about the impact of tobacco smoke inhalation on the adaptive immune system in the human lung.

Animal studies have shown that there is an increase in mDCs [19] with the Langerhans' cell phenotype [23] in the airways 
TABLE 1 Antibodies used for four-colour flow cytometry

\begin{tabular}{|c|c|c|c|}
\hline Antigen & Label & Clone & Company \\
\hline \multicolumn{4}{|l|}{ Lineage cocktail } \\
\hline CD3 & FITC & SK7 & BD Biosciences ${ }^{\#}$ \\
\hline CD14 & FITC & МфP 9 & BD Biosciences ${ }^{\#}$ \\
\hline CD16 & FITC & $3 G 8$ & BD Biosciences ${ }^{\#}$ \\
\hline CD19 & FITC & SJ25C1 & BD Biosciences" \\
\hline CD20 & FITC & L27 & BD Biosciences ${ }^{\#}$ \\
\hline CD56 & FITC & NCAM16.2 & BD Biosciences ${ }^{\#}$ \\
\hline \multicolumn{4}{|l|}{ Other antibodies } \\
\hline CD1a & PE & $\mathrm{NA} 1 / 34$ & Dako" \\
\hline CD11c & PE & S-HCL-3 & BD Biosciences ${ }^{\#}$ \\
\hline Langerin & PE & DCGM4 & Beckmann Coulter \\
\hline HLA-DR & PerCP & L243 & BD Biosciences ${ }^{\#}$ \\
\hline BDCA-1 & APC & AD5-8E7 & Miltenyi Biotec ${ }^{\S}$ \\
\hline BDCA-3 & APC & AD5-14H12 & Miltenyi Biotec ${ }^{\S}$ \\
\hline BDCA-4 & APC & AD5-17F6 & Miltenyi Biotec ${ }^{\S}$ \\
\hline CD11c & APC & S-HCL-3 & BD Biosciences ${ }^{\#}$ \\
\hline CD80 & APC & MEM-233 & ImmunoTools ${ }^{f}$ \\
\hline CD83 & APC & HB15e & Invitrogen \#\# \\
\hline CD86 & APC & BU63 & Invitrogen \#\# \\
\hline CCR5 & APC & $2 \mathrm{D} 7$ & BD Biosciences ${ }^{\#}$ \\
\hline CCR7 & APC & 150503 & R\&D Systems" \\
\hline MMR (CD206) & APC & 19.2 & BD Biosciences ${ }^{\#}$ \\
\hline
\end{tabular}

HLA: human leukocyte antigen; BDCA: blood dendritic cell antigen; MMR: macrophage mannose receptor; FITC: fluorescein isothiocyanate; PE: phycoerythrin; PerCP: peridinin chlorophyll protein; APC: allophycocyanin; CCR: CC chemokine receptor. ${ }^{\#}$ : Heidelberg, Germany; ": Glostrup, Denmark; ${ }^{+}$: Krefeld,

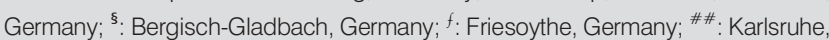
Germany; "थ: Minneapolis, MN, USA.

following chronic tobacco smoke exposure. Human studies with chronic cigarette smokers have confirmed these findings [13-15]. The highest numbers of mDCs with a Langerhans' cell phenotype are found in the airways of smokers with COPD [6] or PLCH [16]. The present study is the first to demonstrate that acute tobacco smoke exposure leads to immediate recruitment of mDCs into human airways in vivo. This recruitment was selective, since there was no increase in $\mathrm{pDCs}$ or other immune cells (such as lymphocytes, granulocytes or macrophages) in BALF directly following tobacco smoke exposure. It should be noted that other particles, such as allergens or infectious agents, do not lead to selective recruitment of this DC subpopulation into human airways $[17,18]$. Therefore, tobacco smoke appears to be a unique stimulus for $\mathrm{mDC}$ recruitment.

It is remarkable that recruitment of $\mathrm{mDC}$ into the airways could already be identified within $1 \mathrm{~h}$ following a 4 -h period of tobacco smoke exposure. This finding is in line with data from MCWILLIAM et al. [22], who described a very rapid influx of DCs into the airways of rats, peaking as soon as $2 \mathrm{~h}$ following antigen challenge. This influx of DCs preceded the influx of neutrophils, thus challenging the classical concept that neutrophils appear in the airways prior to cells of the adaptive immune system following antigen exposure [22]. Therefore, the studies of MCWILLIAM et al. [22] indicated that DC
TABLE 2 Lung function and differential cell counts

\begin{tabular}{lccc} 
& $\begin{array}{c}\text { No smoke } \\
\text { exposure }\end{array}$ & $\begin{array}{c}\text { Acute smoke } \\
\text { exposure }^{\#}\end{array}$ & p-value \\
\hline Lung function & & & \\
FVC \% pred & $103.3(88.1-123.8)$ & $100.5(87.4-123.8)$ & 0.71 \\
FEV1 \% pred & $93.2(75.7-123.8)$ & $95.3(59.3-124.9)$ & 0.74 \\
FEV1/FVC \% & $80.2(64.2-87.9)$ & $81.3(58.5-87.7)$ & 0.80 \\
MEF25\% pred & $51.5(19.3-100.1)$ & $55.2(11.9-100.9)$ & 0.44 \\
RV/TLC \% & $18.0(9.7-42.8)$ & $20.1(9.3-36.5)$ & 0.59 \\
TLC \% pred & $98.5(84.8-115.9)$ & $97.0(72.0-123.4)$ & 0.78 \\
Blood cell counts & & & \\
Leukocytes 10 cells·mL ${ }^{-1}$ & $7.5(4.2-10.8)$ & $6.6(4.2-10.0)$ & 0.35 \\
Monocytes \% & $9.2(6.3-12.9)$ & $8.2(4.8-9.9)$ & $<0.01$ \\
Lymphocytes \% $_{\text {Neutrophils \% }}$ & $34.5(19.7-53.0)$ & $30.8(16.5-60.0)$ & 0.47 \\
Eosinophils \% & $52.2(31.6-63.1)$ & $57.7(32.0-73.5)$ & 0.06 \\
BALF cell counts & $3.3(0.5-9.5)$ & $2.1(0.2-9.3)$ & $<0.05$ \\
Recovery mL & & & \\
Leukocytes 10 ${ }^{6}$ cells·mL ${ }^{-1}$ & $0.12(0.02-0.46)$ & $0.11(0.03-0.29)$ & 0.61 \\
Macrophages \% & $96.0(89.2-98.6)$ & $95.7(85.4-99.4)$ & 0.27 \\
Lymphocytes \% $_{\text {Neutrophils \% }}^{2.4(0.8-6.8)}$ & $3.2(0.4-10.8)$ & 0.09 \\
Eosinophils \% & $0.9(0.0-3.8)$ & $1.1(0.0-4.0)$ & 0.48 \\
\hline & $0.3(0.0-1.0)$ & $0.3(0.0-3.0)$ & 0.43 \\
\hline
\end{tabular}

Data are presented as median (range) $(n=16)$; cell subpopulations are given as a percentage of total leukocytes. FVC: forced vital capacity; \% pred: \% predicted; FEV1: forced expiratory volume in $1 \mathrm{~s}$; MEF25: mean expiratory flow when $75 \%$ of the FVC has been exhaled; RV: residual volume; TLC: total lung capacity; BALF: bronchoalveolar lavage fluid. ${ }^{\#}$ : for $4 \mathrm{~h}$; $"$ : peripheral blood.

\section{TABLE 3 Dendritic cell (DC) counts and chemokine concentrations}

$\begin{array}{lc}\begin{array}{l}\text { No smoke } \\ \text { exposure }^{\#}\end{array} & \begin{array}{c}\text { Acute smoke } \\ \text { exposure }^{\#}\end{array}\end{array}$

\section{Blood DCs}

mDCs \% leukocytes $0.18(0.07-0.37) \quad 0.15(0.07-0.32)<0.05$ $\mathrm{mDCs}$ cells $\cdot \mathrm{mL}^{-1} \quad 13434(4368-30744) \quad 8456(4128-29248) \quad<0.05$ pDCs \% leukocytes $0.21(0.11-0.33) \quad 0.23(0.10-0.36) \quad 0.42$ pDCs cells $\cdot \mathrm{mL}^{-1} \quad 14854(6864-21000) \quad 14380(6490-25404) \quad 0.96$

\section{BALF DCs} $\mathrm{mDCs}$ cells $\cdot \mathrm{mL}^{-1} \quad 685(110-4830) \quad 1494(230-4500) \quad<0.05$ pDCs \% leukocytes $0.07(0.00-0.22) \quad 0.06(0.01-0.35) \quad 0.30$ $\mathrm{pDCs}$ cells $\cdot \mathrm{mL}^{-1} \quad 42(0-401) \quad 100(10-228) \quad 0.26$

BALF chemokines

$\begin{array}{lccc}\mathrm{CCL} 20 \mathrm{pg} \cdot \mathrm{mL}^{-1} & 13.3(4.0-121.5) & 12.3(6.3-49.2) & 0.87 \\ \mathrm{CXCL} 10 \mathrm{pg} \cdot \mathrm{mL}^{-1} & 10.3(8.0-52.8) & 14.2(8.0-44.9) & 0.75\end{array}$

Data are presented as median (range) $(n=16)$. mDC: myeloid DC; $\mathrm{pDC}$ plasmacytoid DC; BALF: bronchoalveolar lavage fluid; CCL: CC chemokine ligand; CXCL: CXC chemokine ligand. ${ }^{\#}$ : for $4 \mathrm{~h}$. 

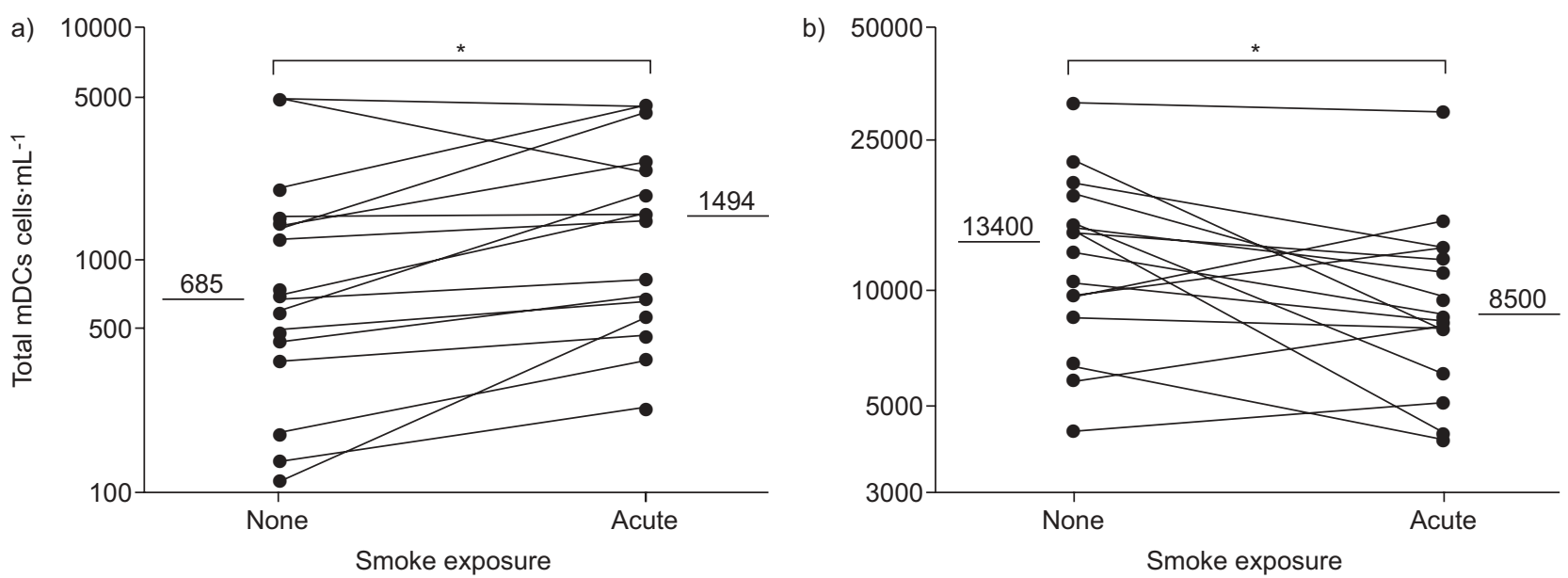

FIGURE 2. Total myeloid dendritic cell (mDC) counts in: a) bronchoalveolar lavage fluid; and b) peripheral blood following $4 \mathrm{~h}$ of nonsmoking and $4 \mathrm{~h}$ of acute smoke exposure. Individual participants are shown $(n=16)$. Horizontal bars represent medians. *: $p<0.05$

recruitment is an integral part of the early phase of the immune response in the airways. However, studies confirming such early DC recruitment in human airways have been lacking. By demonstrating the immediate and selective recruitment of mDCs into the airways directly following acute smoke exposure, the present study confirms the postulate of McWilliam et al. [22] in humans for the first time.

The mechanisms leading to the recruitment of mDCs following tobacco smoke exposure remain unclear. It should be noted that there was no increase in the well-established $\mathrm{mDC}$ attracting chemokine CCL20 [6] in BALF directly following acute smoke exposure. This finding suggests that other mechanisms may lead to the recruitment of mDCs into the airways following acute smoke exposure. However, it should be emphasised that unchanged CCL20 concentrations following acute smoke exposure do not exclude any involvement of

\begin{tabular}{lccc} 
TABLE 4 & $\begin{array}{l}\text { Surface molecule expression on myeloid } \\
\text { dendritic cells (mDC) } \\
\text { fluid }\end{array}$ & & \\
& No smoke exposure & & \\
& Acute smoke exposure & p-value \\
\hline CD1a & $86.9(60.6-93.1)$ & $88.8(76.7-93.8)$ & 0.09 \\
Langerin & $70.8(45.8-83.8)$ & $71.0(53.6-87.1)$ & 0.22 \\
BDCA-1 & $87.7(65.3-93.7)$ & $90.2(64.1-96.0)$ & $<0.05$ \\
BDCA-3 & $73.9(49.9-89.6)$ & $74.6(52.9-89.4)$ & 0.45 \\
BDCA-4 & $38.1(9.1-68.1)$ & $47.0(22.4-67.2)$ & $<0.05$ \\
CD80 & $72.0(48.6-83.4)$ & $73.4(42.6-83.3)$ & 0.29 \\
CD83 & $23.3(11.6-42.8)$ & $20.3(10.0-45.3)$ & 0.91 \\
CD86 & $89.5(66.2-97.6)$ & $91.0(38.5-98.1)$ & 0.39 \\
MMR & $70.9(28.6-85.8)$ & $76.6(39.4-83.1)$ & 0.10 \\
CCR5 & $30.2(9.9-62.2)$ & $27.7(3.6-50.2)$ & $<0.05$ \\
CCR7 & $7.3(3.2-15.2)$ & $5.9(1.1-13.2)$ & 0.41 \\
\hline
\end{tabular}

Data are presented as median (range) percentage of mDCs $(n=16)$. BDCA: blood dendritic cell antigen; MMR: macrophage mannose receptor; CCR: CC chemokine receptor. ${ }^{*}$ : for $4 \mathrm{~h}$. this chemokine in tobacco-smoke-induced inflammation and $\mathrm{mDC}$ recruitment. On the one hand, BALF CCL20 concentrations could already be increased due to the underlying chronic smoke exposure of the participants. On the other hand, the BALF concentrations of this chemokine could increase several hours or days after acute smoke exposure. Therefore, the chosen time-point (directly following smoke exposure) might be too early to observe a smoke-induced increase in this chemokine.

In a previous study, a strong increase was found in the expression of the surface molecules BDCA-1 (CD1c) and BDCA-4 (neuropilin-1) on airway mDCs of chronic cigarette smokers [15]. In the present study, the strong expression of these surface molecules on the mDCs of smokers was confirmed. In addition, it was shown that acute smoke exposure was associated with a further increase in the expression of BDCA- 1 and BDCA-4 on airway mDCs. Thus the findings of the previous and current studies are consistent and point to a specific effect of tobbaco smoke on the BDCA expression profile of human airway mDCs. The functional significance of these findings is currently unknown, and further studies are required in order to define the specific roles of BDCA-1 and BDCA-4 in smoke-related lung diseases.

It should be noted that acute tobacco smoke exposure did not result in increased expression of the maturity marker CD83 on airway mDCs. In a previous study comparing chronic smokers with never-smokers, there was even a trend towards a decrease in CD83 expression on airway mDCs in chronic smokers [15]. These findings are in line with recent reports showing decreased numbers of CD83-expressing cells in the airways of smokers with asthma [24] or COPD [25]. There are two possible scenarios that might explain these observations. On the one hand, decreased CD83 expression on airway mDCs might simply reflect the influx of immature mDCs into the airways. This has been suggested in a study exploring the effects of allergen challenge on airway DCs in patients with asthma [17]. On the other hand, it has been hypothesised that DC maturation may be specifically attenuated by tobacco smoke [26]. Indeed, cigarette smoke extracts [27], as well as 
sputa from patients with COPD [28], have been shown to suppress DC maturation in vitro. The increase in airway $\mathrm{mDC}$ number and the parallel decrease in peripheral blood mDC number might support the first hypothesis (influx of immature DCs). However, the downregulation of the lung homing receptor CCR5 on mDCs following acute smoke exposure is compatible with a more mature $\mathrm{mDC}$ phenotype. In addition, acute smoke exposure led to an increase in the expression of BDCA-4, a surface molecule completely absent on immature blood mDCs, on airway mDCs [15]. Finally, the trend towards increased expression of the MMR does not necessarily point to a more immature phenotype of the DCs following smoke exposure, since there is no correlation between expression of MMR and CD83 on human BALF mDCs [15]. Thus the issue regarding whether or not the absence of an increase in CD83 expression on airway $\mathrm{mDC}$ following tobacco smoke exposure reflects an influx of immature DCs or a partial inhibition of $\mathrm{mDC}$ maturation remains open.

The downregulation of the lung homing chemokine receptor CCR5 [29] following acute smoke exposure, which was also found in a previous study in chronic smokers [15], is compatible with the idea that $\mathrm{mDCs}$ mature after infiltration of the airways and after antigen uptake. In contrast, the chemokine receptor CCR7, which is essential for the migration of mDCs to the draining lymph nodes [30-32], was not upregulated. In the previous study comparing chronic smokers with neversmokers, there was even decreased expression of CCR7 on airway mDCs in chronic smokers [15]. Thus cigarette smoke appears to suppress upregulation of CCR7 on mDCs, which would be expected during a normal maturation process after antigen uptake [33]. In an in vitro study of VASSALLO et al. [27] using human-monocyte-derived DCs, cigarette smoke extracts (but not nicotine alone) suppressed the maturation-associated expression of CCR7. It should be noted that cigarette smoke extracts did not alter the low-level expression of CCR7 on immature DCs, but inhibited the lipopolysaccharide-induced upregulation of CCR7 in a dose-dependent fashion [27]. Therefore, given the essential role of CCR7 for the migration of mDCs to mediastinal lymph nodes [30-32], cigarette smoke could reduce the migratory potential of maturing mDCs. This might have pathogenetic implications for diseases such as COPD [6] and PLCH [16, 34].

However, this requires further study and should, therefore, be discussed with caution. For instance, different levels of CCR7 expression were measured in the previous [15] and current study, although the same antibody clone (clone 150503) from the same manufacturer (R\&D Systems, Minneapolis, MN, USA) with the same label (allophycocyanin) was used. The only difference between the studies was that different batches of this antibody were used. It might, therefore, be speculated that the allophycocyanin-labelling of the antibodies differed between the two batches. The general conclusions of the studies regarding CCR7 are not affected by this methodological issue since all of the patients in one study were analysed with the same batch of the antibody. However, the difference in the general CCR7 expression level reveals that there is currently no established expression level of CCR7 on airway $\mathrm{mDC}$ in human BALF. In addition, the expression of CCR7 on mDCs is known to be strongly dependent upon the compartment under study (BALF, lung parenchyma or mediastinal lymph nodes). Therefore, further studies on the expression and functional role of CCR7 on DCs in human airways are required in order to reach more definite conclusions.

The present study has two major limitations. First, the effects of acute tobbaco smoke exposure on airway DCs were investigated in chronic smokers, but not in never-smokers. Thus the results were confounded by pre-existing smokeinduced changes in the airways. In order to fully understand the impact of tobacco smoke on airway DCs in humans, it would be desirable to test the impact of acute smoke exposure on airway DCs in tobacco-smoke-naive airways. However, acute smoke exposure in nonsmoking individuals was considered ethically inappropriate and was, therefore, rejected. Secondly, the trafficking of airway DCs to the mediastinal lymph nodes following acute smoke exposure was not studied. This would be of interest in elucidating the functional consequences of the changes in the number and surface molecule expression of airway mDCs. However, in order to study the trafficking of airway DCs to the mediastinal lymph nodes in humans, it would be necessary to not only mark airway DCs before smoke exposure but also surgically obtain mediastinal lymph nodes after smoke exposure. These procedures were considered ethically unacceptable.

In conclusion, the present study shows, for the first time, immediate and selective recruitment of mDCs into human airways and specific modulation of several function-associated surface molecules on mDCs following acute tobacco smoke exposure in vivo. These data form the basis for future studies on the role of the adaptive immune system in tobacco smokerelated lung diseases.

\section{SUPPORT STATEMENT}

This study was supported by the German Research Foundation (Bonn, Germany; grant LO 1145/2-2).

\section{CLINICAL TRIAL}

This study is registered at ClinicalTrials.gov (trial number NCT00740896).

\section{STATEMENT OF INTEREST}

None declared.

\section{ACKNOWLEDGEMENTS}

We thank P. Thamm, J. Brandt, G. Fastnacht and C. Beil (all Dept of Pneumology, University of Rostock, Rostock, Germany) for excellent technical assistance.

\section{REFERENCES}

1 Shahab L, Jarvis MJ, Britton J, et al. Prevalence, diagnosis and relation to tobacco dependence of chronic obstructive pulmonary disease in a nationally representative population sample. Thorax 2006; 61: 1043-1047.

2 Rabe KF, Hurd S, Anzueto A, et al. Global strategy for the diagnosis, management, and prevention of chronic obstructive pulmonary disease. GOLD executive summary. Am J Respir Crit Care Med 2007; 176: 532-555.

3 Vassallo R, Ryu JH, Colby TV, et al. Pulmonary Langerhans'-cell histiocytosis. N Engl J Med 2000; 342: 1969-1978.

4 Tazi A. Adult pulmonary Langerhans' cell histiocytosis. Eur Respir J 2006; 27: 1272-1285. 
5 Cosio MG, Saetta M, Agusti A. Immunologic aspects of chronic obstructive pulmonary disease. N Engl J Med 2009; 360: 2445-2454.

6 Demedts IK, Bracke KR, Van Pottelberge G, et al. Accumulation of dendritic cells and increased CCL20 levels in the airways of patients with chronic obstructive pulmonary disease. Am J Respir Crit Care Med 2007; 175: 998-1005.

7 Hogg JC. Pathophysiology of airflow limitation in chronic obstructive pulmonary disease. Lancet 2004; 364: 709-721.

8 Tsoumakidou M, Demedts IK, Brusselle GG, et al. Dendritic cells in chronic obstructive pulmonary disease: new players in an old game. Am J Respir Crit Care Med 2008; 177: 1180-1186.

9 Brusselle GG, Demoor T, Bracke KR, et al. Lymphoid follicles in (very) severe COPD: beneficial or harmful? Eur Respir J 2009; 34: 219-230.

10 Vermaelen K, Pauwels R. Pulmonary dendritic cells. Am J Respir Crit Care Med 2005; 172: 530-551.

11 Blank F, Rothen-Rutishauser B, Gehr P. Dendritic cells and macrophages form a transepithelial network against foreign particulate antigens. Am J Respir Cell Mol Biol 2007; 36: 669-677.

12 Veres TZ, Shevchenko M, Krasteva G, et al. Dendritic cell-nerve clusters are sites of $\mathrm{T}$ cell proliferation in allergic airway inflammation. Am J Pathol 2009; 174: 808-817.

13 Casolaro MA, Bernaudin JF, Saltini C, et al. Accumulation of Langerhans' cells on the epithelial surface of the lower respiratory tract in normal subjects in association with cigarette smoking. Am Rev Respir Dis 1988; 137: 406-411.

14 Soler P, Moreau A, Basset F, et al. Cigarette smoking-induced changes in the number and differentiated state of pulmonary dendritic cells/Langerhans cells. Am Rev Respir Dis 1989; 139: 1112-1117.

15 Bratke K, Klug M, Bier A, et al. Function-associated surface molecules on airway dendritic cells in cigarette smokers. Am J Respir Cell Mol Biol 2008; 38: 655-660.

16 Caminati A, Harari S. Smoking-related interstitial pneumonias and pulmonary Langerhans cell histiocytosis. Proc Am Thorac Soc 2006; 3: 299-306.

17 Bratke K, Lommatzsch M, Julius P, et al. Dendritic cell subsets in human bronchoalveolar lavage fluid after segmental allergen challenge. Thorax 2007; 62: 168-175.

18 Lommatzsch M, Bratke K, Bier A, et al. Airway dendritic cell phenotypes in inflammatory diseases of the human lung. Eur Respir J 2007; 30: 878-886.

19 D'Hulst AI, Vermaelen KY, Brusselle GG, et al. Time course of cigarette smoke-induced pulmonary inflammation in mice. Eur Respir J 2005; 26: 204-213.
20 van der Vaart H, Postma DS, Timens W, et al. Acute effects of cigarette smoke on inflammation and oxidative stress: a review. Thorax 2004; 59: 713-721.

21 van der Vaart H, Postma DS, Timens W, et al. Acute effects of cigarette smoking on inflammation in healthy intermittent smokers. Respir Res 2005; 6: 22.

22 McWilliam AS, Nelson D, Thomas JA, et al. Rapid dendritic cell recruitment is a hallmark of the acute inflammatory response at mucosal surfaces. J Exp Med 1994; 179: 1331-1336.

23 Zeid NA, Muller HK. Tobacco smoke induced lung granulomas and tumors: association with pulmonary Langerhans cells. Pathology 1995; 27: 247-254.

24 Tsoumakidou M, Elston W, Zhu J, et al. Cigarette smoking alters bronchial mucosal immunity in asthma. Am J Respir Crit Care Med 2007; 175: 919-925.

25 Tsoumakidou M, Koutsopoulos AV, Tzanakis N, et al. Decreased small airway and alveolar CD83+ dendritic cells in chronic obstructive pulmonary disease. Chest 2009; 136: 726-733.

26 Tsoumakidou M, Jeffery PK. Dendritic cell maturity and obstructive airway disease. Am J Respir Crit Care Med 2007; 176: 833.

27 Vassallo R, Tamada K, Lau JS, et al. Cigarette smoke extract suppresses human dendritic cell function leading to preferential induction of Th-2 priming. J Immunol 2005; 175: 2684-2691.

28 Roghanian A, Drost EM, MacNee W, et al. Inflammatory lung secretions inhibit dendritic cell maturation and function via neutrophil elastase. Am J Respir Crit Care Med 2006; 174: 1189-1198.

29 Sallusto F, Lanzavecchia A. Understanding dendritic cell and Tlymphocyte traffic through the analysis of chemokine receptor expression. Immunol Rev 2000; 177: 134-140.

30 Randolph GJ, Angeli V, Swartz MA. Dendritic-cell trafficking to lymph nodes through lymphatic vessels. Nat Rev Immunol 2005; 5: 617-628.

31 Jakubzick C, Tacke F, Llodra J, et al. Modulation of dendritic cell trafficking to and from the airways. I Immunol 2006; 176: 35783584.

32 Sanchez-Sanchez N, Riol-Blanco L, Rodriguez-Fernandez JL. The multiple personalities of the chemokine receptor CCR7 in dendritic cells. J Immunol 2006; 176: 5153-5159.

33 Forster R, Schubel A, Breitfeld D, et al. CCR7 coordinates the primary immune response by establishing functional microenvironments in secondary lymphoid organs. Cell 1999; 99: 23-33.

34 Annels NE, Da Costa CE, Prins FA, et al. Aberrant chemokine receptor expression and chemokine production by Langerhans cells underlies the pathogenesis of Langerhans cell histiocytosis. J Exp Med 2003; 197: 1385-1390. 